\title{
OPEN A highly stable, nanotube-enhanced, CMOS-MEMS thermal emitter for mid-IR gas sensing
}

\author{
Daniel Popa ${ }^{1 凶}$, Richard Hopper ${ }^{1}$, Syed Zeeshan Ali ${ }^{2}$, Matthew Thomas Cole ${ }^{3}$, Ye Fan $^{1}$, \\ Vlad-Petru Veigang-Radulescu ${ }^{1}$, Rohit Chikkaraddy ${ }^{4}$, Jayakrupakar Nallala ${ }^{5}$, Yuxin Xing ${ }^{6}$, \\ Jack Alexander-Webber ${ }^{1}$, Stephan Hofmann ${ }^{1}$, Andrea De Luca ${ }^{2}$, Julian William Gardner ${ }^{6}$ \& \\ Florin Udrea ${ }^{1}$
}

The gas sensor market is growing fast, driven by many socioeconomic and industrial factors. Mid-infrared (MIR) gas sensors offer excellent performance for an increasing number of sensing applications in healthcare, smart homes, and the automotive sector. Having access to low-cost, miniaturized, energy efficient light sources is of critical importance for the monolithic integration of MIR sensors. Here, we present an on-chip broadband thermal MIR source fabricated by combining a complementary metal oxide semiconductor (CMOS) micro-hotplate with a dielectric-encapsulated carbon nanotube (CNT) blackbody layer. The micro-hotplate was used during fabrication as a microreactor to facilitate high temperature $\left(>700^{\circ} \mathrm{C}\right)$ growth of the $\mathrm{CNT}$ layer and also for post-growth thermal annealing. We demonstrate, for the first time, stable extended operation in air of devices with a dielectric-encapsulated CNT layer at heater temperatures above $600^{\circ} \mathrm{C}$. The demonstrated devices exhibit almost unitary emissivity across the entire MIR spectrum, offering an ideal solution for lowcost, highly-integrated MIR spectroscopy for the Internet of Things.

Gas sensors are at the center of increasing research and development efforts, driven by many scientific, industrial and commercial applications ${ }^{1}$. These include the monitoring of environmental pollutants from deforestation ${ }^{2}$, vehicles and industry ${ }^{3}$ and also air quality within buildings ${ }^{4}$. There is an increased awareness of the impact of air pollution on human health ${ }^{3}$, leading to a rise in demand for low cost, accessible, compact, and readily deployable air quality monitoring ${ }^{5}$. To sustain the emerging global demand, gas sensors must meet a suitable and challenging balance between performance and $\operatorname{cost}^{1}$. In addition to being economically viable, an increasing number of sensors have stringent power and volume constraints ${ }^{1}$, e.g. those deployed within the Internet of Things (IoT) ${ }^{6}$ and in mobile platforms ${ }^{7}$. These requirements motivate researchers to explore novel materials, designs and technologies to achieve miniaturization, monolithic integration of components, low cost, reduced power consumption and manufacturability ${ }^{1}$.

Amongst the various different sensing technologies, optical gas sensors offer several advantages in terms of selectivity and long-term operational stability ${ }^{1}$. Notably, nondispersive infrared (NDIR) sensors currently dominate the carbon dioxide $\left(\mathrm{CO}_{2}\right)$ gas sensor market, and also serve many other applications ${ }^{8}$. However, despite their inherent advantages (e.g., for spectroscopic sensing), NDIR gas sensors are currently mostly employed for the detection of single analytes, or a few species at the same time. A limit to wider adoption has been availability of miniaturised broadband MIR light sources which are low cost and optically efficient (arguably the core of an optical gas sensor $)^{1}$. Bulb based thermal sources have traditionally been used but are fragile, bulky and have limited optical efficiency at wavelengths above $5 \mu \mathrm{m}$. Light-emitting diodes (LED) offer improved integration and reliability but are costlier to fabricate due to the use of specialist III-V semiconductor technologies ${ }^{9}$.

\footnotetext{
${ }^{1}$ Department of Engineering, University of Cambridge, Cambridge CB3 OFA, UK. ${ }^{2}$ Flusso Limited, Cambridge CB4 $0 D L$, UK. ${ }^{3}$ Department of Electronic and Electrical Engineering, University of Bath, Bath BA2 7AY, UK. ${ }^{4}$ Department of Physics, University of Cambridge, Cambridge CB3 OHE, UK. ${ }^{5}$ Biomedical Physics, School of Physics and Astronomy, University of Exeter, Exeter EX4 4QL, UK. ${ }^{6}$ School of Engineering, University of Warwick, Coventry CV4 7AL, UK. ${ }^{\boxplus}$ email: dp387@cam.ac.uk
} 

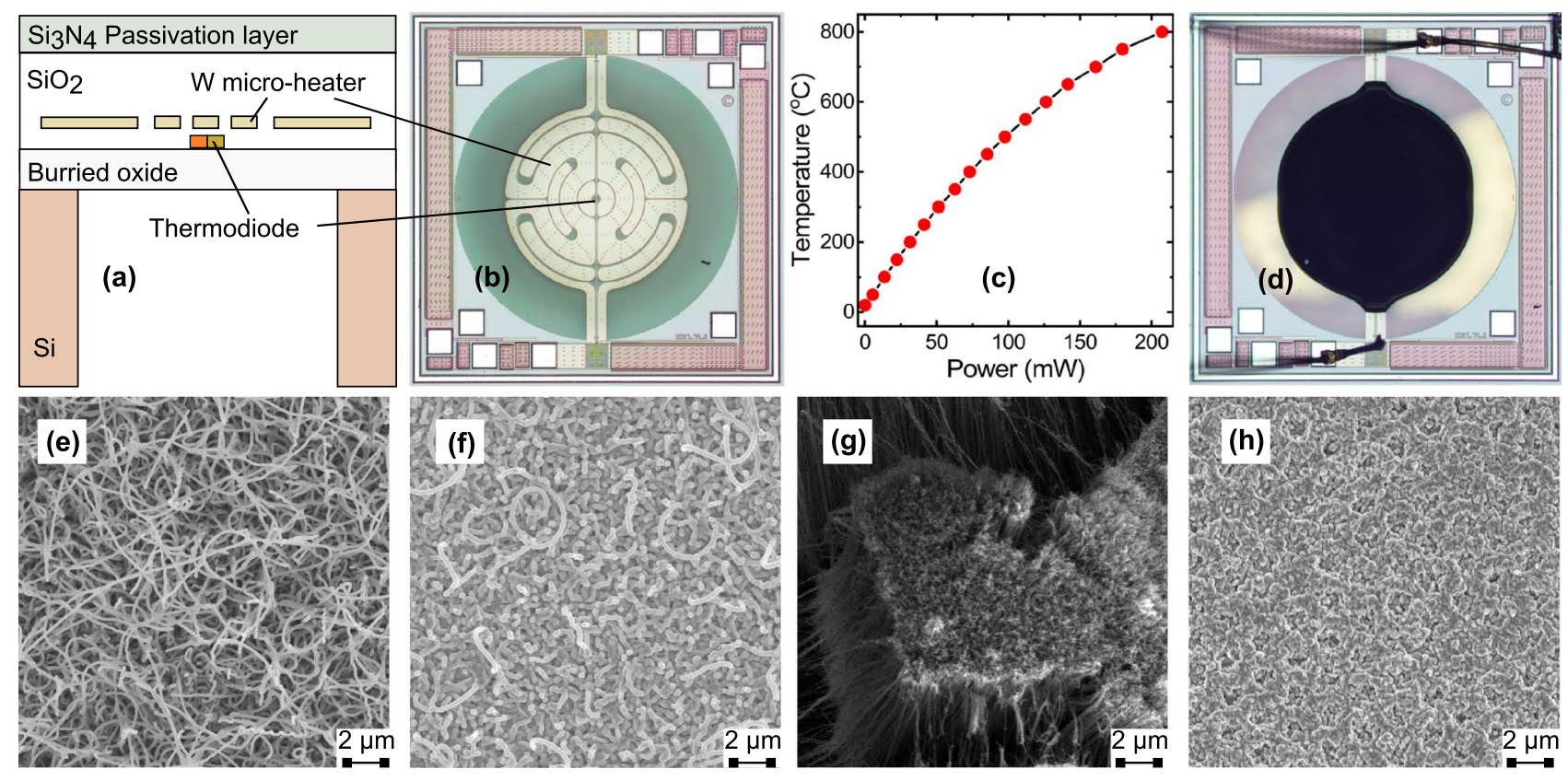

Figure 1. Device fabrication. (a) Micro-hotplate cross-section (not to scale) employing a tungsten (W) heating element embedded into a $\sim 5 \mu \mathrm{m}$ thick silicon dioxide $\left(\mathrm{SiO}_{2}\right)$ membrane formed by deep reactive ion etching. (b) Optical image of the micro-hotplate, showing a multi-ring designed heating element surrounded by the membrane. Chip size $=1.76 \mathrm{~mm} \times 1.76 \mathrm{~mm}$. (c) Micro-heater temperature as a function of power consumption. (d) The active heating element serves three purposes: (i) localised in-situ heating (micro-reactor) during the CNT growth process; (ii) employed as thermal profiler (adlayer flashing) for self-annealing; and (iii) used to generate IR emission during device operation (typically at temperatures of $300-600{ }^{\circ} \mathrm{C}$ ). (e) Typical SEM image of spaghetti-like CNT structure grown by an in-situ CVD process using acetylene $\left(\mathrm{C}_{2} \mathrm{H}_{2}\right)$ blended with ammonia $\left(\mathrm{NH}_{3}\right)$ over an iron $(\mathrm{Fe})$ catalyst, and $(\mathbf{f})$ their SEM image after $\sim 50 \mathrm{~nm}$ alumina $\left(\mathrm{Al}_{2} \mathrm{O}_{3}\right)$ encapsulation. (e) SEM image of denser, more aligned CNTs synthesised by mixing $\mathrm{C}_{2} \mathrm{H}_{2}$ with hydrogen $\left(\mathrm{H}_{2}\right)$, and (h) their SEM image after encapsulation.

Exploiting standard complementary metal-oxide semiconductor (CMOS) processes is an attractive way to fabricate low-cost integrated thermal MIR sources and detectors, and has led to many innovative micro-electromechanical system (MEMS) based devices ${ }^{1,10}$. Various techniques have been proposed to enhance the emissivity/ absorptivity $^{1}$ of CMOS-MEMS thermal devices, including the use of carbon nanotubes (CNTs) adlayers offering near unit broadband emissivity ${ }^{11,12}$, or plasmonic metamaterials for specific MIR bands ${ }^{13,14}$. Multi-species spectroscopic detection requires the MIR source to operate at an ensemble of target MIR wavebands, making the overall CNT broadband emission enhancement ${ }^{11,12}$ attractive for spectroscopy ${ }^{1}$. However, despite their blackbody-like advantages ${ }^{15,16}$, thus far most research has observed such CNT, and in general all graphitic nanocarbon adlayers, burning off in air when operated at temperatures above $400{ }^{\circ} \mathrm{C}^{17,18}$. This poses a limit (optical emission and operational stability) to their integration into CMOS MEMS micro-hotplate MIR sources, which are typically operated at these temperatures ${ }^{19}$. Although the use of an inert gas can be used to prevent CNT burn-off, this requires the use of specialist hermetically sealed ceramic or metal packages, which can significantly impact cost ${ }^{20,21}$.

Here, we present a solid-state approach based on a dielectric-encapsulation method that enables the longterm operational stability of CNT-coated thermal emitters. We show that alumina $\left(\mathrm{Al}_{2} \mathrm{O}_{3}\right)$-encapsulated CNTs grown on a MEMS micro-hotplate can withstand temperatures in excess of $800{ }^{\circ} \mathrm{C}$ when operated in air. The encapsulated CNT adlayer has an emissivity close to unity (a $\sim 8$-fold increase with a reference to a standard MEMS device) and demonstrates stable operation at $600{ }^{\circ} \mathrm{C}$ for 10 days. The work paves the way for encapsulation techniques to be more widely applied to temperature and air sensitive nanomaterials, allowing them to operate stably in air, well above their normal temperature threshold.

\section{Results}

For our experiment, we use an in-house designed micro-hotplate fabricated at a commercial foundry. The microhotplate cross-section is shown in Fig. 1a and consists of a multi-ring resistive tungsten (W) heating element ( $800 \mu \mathrm{m}$ diameter) embedded within a $\sim 5 \mu \mathrm{m}$ thick silicon dioxide $\left(\mathrm{SiO}_{2}\right)$ membrane $(1200 \mu \mathrm{m}$ diameter $)$, to ensure low direct current (DC) power consumption ${ }^{19}$. W was chosen as the heating element and interconnection metal due to its superior resistance toward electromigration and higher glass transition temperature when compared to doped poly-silicon or aluminum ${ }^{10,19}$. The micro-hotplate is built in a CMOS-SOI (silicon on insulator) technology and features a monolithically integrated, monocrystalline silicon thermodiode which can operate linearly with high accuracy at record temperatures up to $600{ }^{\circ} \mathrm{C}^{22}$. The thermodiode can be used as an accurate temperature sensor when the micro-hotplate is used as an IR emitter and when calibrated allows resolutions below $0.5^{\circ} \mathrm{C}$. For the growth of the $\mathrm{CNTs}$ and operation above $600{ }^{\circ} \mathrm{C}$ it is however preferred to use 
the $\mathrm{W}$ heater as a resistive temperature detector (RTD). W has a large and a stable temperature coefficient of resistance (TCR) $\left(\sim 4.5 \times 10^{-3} \mathrm{~K}^{-1}\right)$ and has been shown to function up to $1000^{\circ} \mathrm{C}$ with relatively high accuracy $\sim 2{ }^{\circ} \mathrm{C}^{19}$. Micro-hotplates can reach temperatures in excess of $700^{\circ} \mathrm{C}$ and have fast thermal transient times $>4 \times$ $10^{4} \mathrm{C} / \mathrm{s}$, enabling voltage-controlled thermal ramps and stable MIR emission with excellent reproducibility at very low $\operatorname{cost}^{10,19}$. An optical image of our fabricated micro-hotplate, showing the heating element surrounded by the membrane is given in Fig. $1 b$.

We use an in-situ chemical vapour deposition (CVD) process to integrate the CNT adlayer ${ }^{11,12}$. CVD processes typically require substrate temperatures in excess of $400{ }^{\circ} \mathrm{C}^{23,24}$, which are not CMOS compatible, as temperature effects can damage the integrated circuitry (e.g. due to accelerated alloying and atomic migration) ${ }^{10}$. Due to the thermal isolation offered by the thin dielectric membrane, our micro-hotplates can easily reach temperatures in excess of $750{ }^{\circ} \mathrm{C}$ (Fig. 1c) in localized "hot zones", without compromising the functionalities of the peripheral CMOS circuitry placed on the chip substrate that are only a few micrometers distant from the hot zone. Hence, our micro-hotplates can be effectively employed as ideal CMOS compatible micro-reactors, allowing viable CNT-CMOS integration at wafer level ${ }^{12}$. In addition, our design allows for relatively low DC power consumption to achieve such high temperatures (e.g. $\sim 100 \mathrm{~mW}$ at $500{ }^{\circ} \mathrm{C}$, as shown in Fig. 1c), which can be further minimized by using modulated driving signals (e.g. $50 \%$ duty cycle in our case). To test the encapsulation efficiency, we used two commonly used process gases, ammonia $\left(\mathrm{NH}_{3}\right)^{12}$ and hydrogen $\left(\mathrm{H}_{2}\right)^{11}$, blended with the same carbon-containing gas [acetylene $\left(\mathrm{C}_{2} \mathrm{H}_{2}\right)$ ], over an iron $(\mathrm{Fe})$ catalyst process. As reported elsewhere in the CNTsynthesis literature, using $\mathrm{NH}_{3}$ tends to give more spaghetti-like bundles of nanotubes ${ }^{12}$, whilst using $\mathrm{H}_{2}$ gives more spatially dense, vertically aligned nanotubes ${ }^{11}$, thus providing us with a comprehensive testbed (impacts of CNT crystallography and morphology) for our experiment. An optical image for a $\mathrm{NH}_{3}: \mathrm{C}_{2} \mathrm{H}_{2}$-synthesised CNT sample is shown in Fig. 1d. Scanning electron microscopy (SEM) inspection confirms the successful growth of both spaghetti-like (Fig. 1e) and vertically aligned (Fig. 1g) nanotube forests.

It is known that CNTs burn in air (typically within minutes) at temperatures above $400{ }^{\circ} \mathrm{C}$, depending on their diameter, number of walls or amount of defects ${ }^{17,18}$, thus hindering their application as thermal emitters. To isolate the air-exposed CNTs from oxidation ${ }^{17,18}$, and create a thermally stable emitter interface, we encapsulate the as-grown CNTs in a $\sim 50 \mathrm{~nm}$ thick atomic layer deposited (ALD) $\mathrm{Al}_{2} \mathrm{O}_{3}$ coating. $\mathrm{Al}_{2} \mathrm{O}_{3}$-based encapsulation guarantees high thermo-mechanical stability, and was shown to have the capability of operation at temperatures above $500{ }^{\circ} \mathrm{C}$, while proving a good barrier for oxygen $\left(\mathrm{O}_{2}\right)$ and water $\left(\mathrm{H}_{2} \mathrm{O}\right)^{25,26}$. Figure 1f,h show the SEM images, after encapsulation, of the CNTs shown in Fig. 1e (spaghetti-like $\mathrm{NH}_{3}: \mathrm{C}_{2} \mathrm{H}_{2}$-synthesised) and g (aligned $\mathrm{H}_{2}: \mathrm{C}_{2} \mathrm{H}_{2}$-synthesised) respectively. In order to improve the thermal stability after encapsulation, we used a device self-annealing ${ }^{26,27}$ process enabled, in our case, by the micro-heaters themselves. Thermal profiles were applied with electro-thermal modulation in $\sim 100{ }^{\circ} \mathrm{C}$ steps with temperatures of up to $\sim 800{ }^{\circ} \mathrm{C}$; this aligned the induced thermo-mechanical stress on the membrane with the operating frequency thereby avoiding membrane breakages.

A typical optical absorption spectrum (see "Methods") for our micro-hotplates, in the 2-14 $\mu \mathrm{m}$ waveband, is shown in Fig. 2a (black line), with an absorption peak of $\sim 85 \%$ at $8.5 \mu \mathrm{m}$; a signature of the Si-O stretching vibrations within the $\mathrm{SiO}_{2}$ membrane ${ }^{28,29}$. In the same figure (red line), a micro-hotplate with an in-situ grown $\mathrm{NH}_{3}: \mathrm{C}_{2} \mathrm{H}_{2}$-synthesised CNT (Fig. 1f) layer exhibits almost $100 \%$ absorption, a behaviour attributed to the CNT layer's blackbody-like nature ${ }^{15,16}$. To study the emission properties of our uncoated, CNT-coated and encapsulated devices, we implemented a proportional integral derivative (PID)-based temperature controller, able to control the self-heating micro-hotplate temperature within $\sim 0.5{ }^{\circ} \mathrm{C}$ resolution. The emission spectra of the self-heated devices was then measured by a MIR spectrometer (Bentham) (Fig. 2b). A representative spectrum, recorded at $600{ }^{\circ} \mathrm{C}$, for an encapsulated $\left(\mathrm{NH}_{3}: \mathrm{C}_{2} \mathrm{H}_{2}\right)$ sample, is shown in Fig. $2 \mathrm{~b}$ (red line), showing a $\sim 8$-fold emission enhancement when compared to an uncoated device operated at the same temperature (black line). We also measured the emission spectra of unencapsulated CNT-coated devices which, as expected, exhibited a rapid ( $\sim$ minutes) decrease in emission at elevated temperature, consistent with CNTs burning off when exposed to air ${ }^{17}$. An example spectrum is shown in Fig. $2 \mathrm{~b}$ (blue line), recorded after $\sim 10 \mathrm{~min}$ of operation at $600{ }^{\circ} \mathrm{C}$. A visual comparison between black and blue lines suggests that most CNTs had burnt off, confirmed by a subsequent visual inspection under a microscope (Fig. 2b, inset).

To investigate the long-term operational stability of our encapsulated devices, we performed stress testing in air under normal room conditions [standard pressure (1010-1020 mbar), temperature $\left(18-21{ }^{\circ} \mathrm{C}\right)$ and relative humidity (30-50\%)] at temperatures up to $900^{\circ} \mathrm{C}$, which are significantly higher than the actual device operating temperature $\left(\sim 500-600{ }^{\circ} \mathrm{C}\right)$. Typical emission spectra, for a device operated continuously at $600{ }^{\circ} \mathrm{C}$ over a 10 day period (recorded periodically every $24 \mathrm{~h}$ ), are presented in Fig. $2 \mathrm{c}$, with $\sim 3 \times 10^{-5}$ standard deviation variation, indicating excellent stability. Optical images, recorded at representative times during the test, are also presented, which show that the encapsulated CNTs are physically unaffected by high operating temperature. Devices with encapsulated in-situ grown CNTs (synthesised in both $\mathrm{H}_{2}$ or $\mathrm{NH}_{3}$ ) were found to have stable and reproducible emission spectra when operated for hours at temperatures of up to $900{ }^{\circ} \mathrm{C}$. An optical image of a sample is shown in the bottom-right corner of Fig. $2 \mathrm{c}$, recorded after $4 \mathrm{~h}$ of operation in air at $850{ }^{\circ} \mathrm{C}$, further showing the physical stability of the CNTs at high temperature. It is worth noting that at such high temperatures some membranes (not the CNTs) failed, due to extreme thermally-induced mechanical stress. Failed devices were analysed by optical and SEM imaging and the failures identified were down to membrane breakage, rather than burn-off of the encapsulated CNT layer which stayed intact, even at the extreme temperatures. Similar membrane breakage at extreme temperatures has been observed with non-coated devices (without the CNT layer). To check the quality of the encapsulated CNTs before and after the 10-day operation test, we characterized them using Raman spectroscopy. Figure 2d (bottom two lines) plots the Raman spectra of the devices presented above. We do not observe any change in the $\mathrm{D}$ peak position, width and intensity, indicating the long-term high-temperature operation does not induce additional defects with respect to the starting material ${ }^{30}$. As a comparison (see top two lines), we also present the Raman spectra for the same test ran with $\mathrm{H}_{2}: \mathrm{C}_{2} \mathrm{H}_{2}$ encapsulated CNTs instead, 

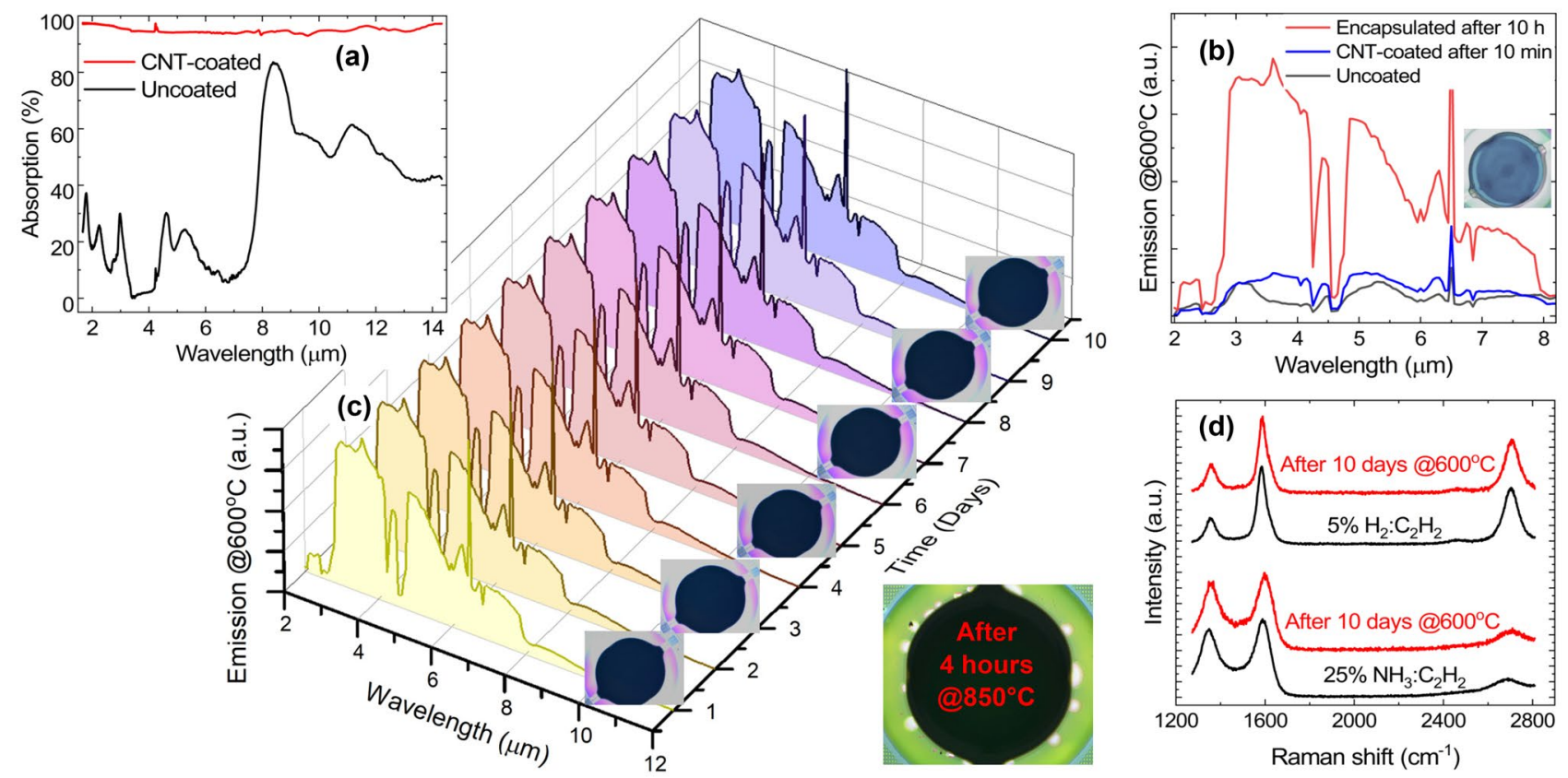

Figure 2. Characterization and stability tests in air. (a) Infrared absorption spectra of an uncoated microhotplate (black line) compared to that of a CNT-coated plate (red line) absorbing almost all light. (b) Emission spectra measured at $600{ }^{\circ} \mathrm{C}$ for an uncoated (black line), CNT-coated (blue line), and encapsulated (red line) micro-hotplate, respectively. CNT-coated devices show a $\sim 8$-fold emission increase when compared to uncoated devices at equivalent temperature, however, unencapsulated CNTs burn-off within minutes, resulting in their emission spectrum (blue line) falling from an initial value close to that of an encapsulated device (red line) to that of an uncoated device (black line). Optical inspection (inset) shows CNTs have almost entirely burned-off. (c) Emission spectra measured at $600{ }^{\circ} \mathrm{C}$ over a 10-day period, for a device with encapsulated $\mathrm{NH}_{3}$ $: \mathrm{C}_{2} \mathrm{H}_{2}$-synthesised CNTs (shown in Fig. 1f). Optical images recorded at respective times show encapsulated $\mathrm{CNTs}$ are not affected by the high temperature operation. An optical image of a test sample after operating at a much higher, $850^{\circ} \mathrm{C}$, temperature for 4 hours is presented in the bottom-right corner, showing the CNTs are intact. (d) (bottom two lines) Raman spectra measured at $532 \mathrm{~nm}$ before and after the operation stability test presented in (c). As a comparison, the top two lines show the Raman spectra measured for the same test (not shown) done with $\mathrm{H}_{2}: \mathrm{C}_{2} \mathrm{H}_{2}$-synthesised CNTs (shown in Fig. 1h) instead, showing both samples remain stable.
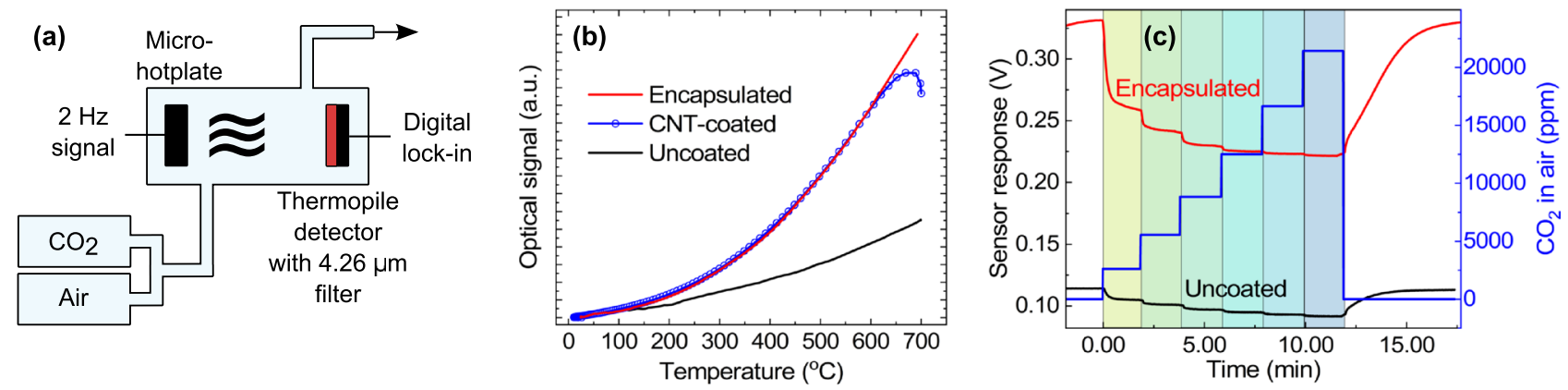

Figure 3. Nondispersive infrared (NDIR) experiment. (a) Schematic of the NDIR gas sensor setup. (b) Relative optical response, recorded by the thermopile at $4.26 \mu \mathrm{m}$, for an uncoated (black line), CNT-coated (dotted blue line), and encapsulated (red line) micro-hotplate respectively. The detected IR emission from the unencapsulated device (dotted blue line) drops rapidly (minutes) at temperatures above $600{ }^{\circ} \mathrm{C}$, consistent with data presented in Fig. 2b. (c) $\mathrm{CO}_{2}$ sensor response voltage with an uncoated (black line), and encapsulated (red line) micro-hotplate respectively. A $\sim 8$-fold increase in relative voltage $\Delta V=V_{0 p p m}-V_{\text {lock-in }}$ can be observed for the encapsulated CNT device.

showing good operational stability for both samples, thus highlighting the broader application potential of our encapsulation technique.

To test the performance of our MIR emitter in an application type setup, samples were benchmarked against an uncoated device using a custom made NDIR gas sensor designed for $\mathrm{CO}_{2}$ detection. A schematic of our experimental setup is shown in Fig. 3a. We use our thermal emitter as a MIR light source, coupled to a singlechannel thermopile detector (Heimann HMS-J21) with integrated $4.26 \mu \mathrm{m}$ filter tuned to the $\mathrm{CO}_{2}$ absorption 
waveband. Both emitter and detector are fitted in a custom gas cell with an optical path length of $4 \mathrm{~cm}$ between emitter and detector. The gas cell is connected to a gas mixing system, enabling the $\mathrm{CO}_{2}$ concentration $\left(c_{\mathrm{CO}_{2}}\right)$ to be controlled by mixing $c_{\mathrm{CO}_{2}}$ at $5 \%$ from a cyclinder with dry air. Our custom-built experimental setup allows $\mathrm{CO}_{2}$ concentrations to be controlled below $100 \mathrm{ppm}$ with a minimum controlled change in concentration of a few ppm. The relative optical signal recorded by the thermopile at room conditions (see "Methods") is shown in Fig. 3b. A CNT $\left(\mathrm{NH}_{3}: \mathrm{C}_{2} \mathrm{H}_{2}\right.$-synthesised)-coated device (dotted blue line) gives a similar response to that of an encapsulated device (red line) to temperatures up to $\sim 60{ }^{\circ} \mathrm{C}$, but the emission level starts to decrease rapidly (within minutes) towards that of a typical uncoated device, confirming the results presented in Fig. 2b. We found no noticeable change in the response of the device encapsulating CNTs (Fig. 3b, red line) after several weeks of operation. Figure $3 \mathrm{c}$ shows the sensor's voltage response with an encapsulated CNT device (red line), compared to an uncoated device (black line) with $c_{\mathrm{CO}_{2}}$ ranging from 0 to $\sim 21,500 \mathrm{ppm}$. The encapsulated CNT device gives a response of $\Delta V=V_{0 p p m}-V_{\text {lock-in }}=111 \mathrm{mV}$ at maximum $\mathrm{CO}_{2}$ concentration, compared to only $22 \mathrm{mV}$ for the uncoated device. The standard deviation of the $\mathrm{CO}_{2}$ measurements is $\sim 0.6 \mathrm{mV}$, which is much less than the amplitude recovered signal post amplification $200-300 \mathrm{mV}$, with the signal-to-noise ratio (SNR) mainly limited by thermal noise. Considering a relative sensitivity defined as $S=\left.\frac{d \Delta V}{d c_{C O}{ }_{2}}\right|_{c_{\mathrm{CO}_{2}}=0}$ and a $\sim 3 \mathrm{~dB}$ measured SNR, we estimate a $\sim 0.12 \mathrm{ppm}$ limit of detection for an encapsulated device compared to $\sim 1 \mathrm{ppm}$ for an uncoated one, consistent with the results in Fig. 2 b.

\section{Discussion}

In conclusion, we demonstrate an efficient CMOS-compatible CNT-encapsulated micro-hotplate-based MIR emitter fabricated on a single $\mathrm{SiO}_{2}$ dielectric membrane, with near unity emissivity, and long-term operational stability. Processing of the chip is simplified by employing standard CMOS tungsten heaters as micro-reactors for precise in-situ CNT growth, allowing scalable integration at waver level. The same heating elements also serve as enablers for versatile self-annealing thermal profiles that can be adapted for easy optimization of various processes. We show stable operation for alumina-encapsulated CNTs up to record temperatures of $900{ }^{\circ} \mathrm{C}$. Employing the emitter in a proof-of-concept optical sensing demonstration, we measured a 8 -fold increase in relative sensitivity to $\mathrm{CO}_{2}$ compared to the use of a conventional MEMS emitter. Our emitter exhibits almost unitary emissivity across the entire MIR band, making it particularly attractive for a variety of low-cost, lowpower and high volume spectroscopic applications in the MIR spectral region.

\section{Methods}

The micro-hotplates were designed in Cadence(C) and fabricated using a commercial $1 \mu \mathrm{m}$ SOI-CMOS process on 6 inch silicon ( $\mathrm{Si}$ ) wafers. The membrane was formed by deep reactive ion etching (DRIE) of a $400 \mu \mathrm{m}$ thick $\mathrm{Si}$ substrate, with the buried $\mathrm{SiO}_{2}$ layer acting as an etch stop. A silicon nitride $\left(\mathrm{Si}_{3} \mathrm{~N}_{4}\right)$ passivation layer shields the membrane from environmental factors, such as humidity ${ }^{31}$.

CNTs were grown by an in-situ thermal CVD of $\mathrm{C}_{2} \mathrm{H}_{2}$ over a Fe catalyst process. The micro-hotplates were coated (ALD) with a $\sim 10 \mathrm{~nm} \mathrm{Al}{ }_{2} \mathrm{O}_{3}$ then sputtered with a 2-4 nm Fe catalyst. The devices [mounted onto TO-type packages connected to a power supply (Keithley 2400)], were then transferred to a custom-build CVD chamber, to grow the CNTs, which was pumped down to a base pressure of $\sim 0.5 \mathrm{mbar}$. The CNT growth process was optimized by a $\sim 0.5{ }^{\circ} \mathrm{C}$ resolution PID-based temperature controller, implemented in LabVIEW@ software, which was set to a $20^{\circ} \mathrm{C} / \mathrm{s}$ heating rate. High purity $\mathrm{NH}_{3}$ or $\mathrm{H}_{2}$ was introduced into the chamber when the microheater reached $\sim 500{ }^{\circ} \mathrm{C}$, and then operated at $725^{\circ} \mathrm{C}$ for $60 \mathrm{~s}$ to form the small catalyst Fe islands. $\mathrm{C}_{2}$ $\mathrm{H}_{2}$ was then introduced through a separate line in a $5 \% \mathrm{H}_{2}: \mathrm{C}_{2} \mathrm{H}_{2}$ or $25 \% \mathrm{NH}_{3}: \mathrm{C}_{2} \mathrm{H}_{2}$ atmosphere respectively, maintained to $\sim 4$ mbar during a $\sim 10$ min growth process. Devices were then introduced into an ALD reactor (Cambridge NanoTech) to deposit $\sim 50 \mathrm{~nm} \mathrm{Al}{ }_{2} \mathrm{O}_{3}$ [using trimethylaluminium (TMA) and water $\left(\mathrm{H}_{2} \mathrm{O}\right)$ as precursors at $200{ }^{\circ} \mathrm{C}$ ] for encapsulation, followed by a self-annealing process at $\sim 400,500,600$, and $700{ }^{\circ} \mathrm{C}$, for 30 min each, respectively.

To obtain the optical absorption (A) spectral profiles, in the wavelength range of $2-14 \mu \mathrm{m}$, transmission (T) and reflection (R) FTIR measurements (normal incidence) were coupled to give $A=1-\mathrm{R}-\mathrm{T}$. The optical aperture of the micro-FTIR system (Agilent Cary 620 FTIR Microscope) was set to image only the heater area of the micro-hotplate. The emission spectral profiles were measured by mounting the devices to a bespoke MIR spectrometer (Bentham) composed by a monochromator (TMc300) connected to a cryogenically-cooled mercury cadmium telluride detector (DH-MTC). Raman spectra were acquired by a Renishaw inVia Raman microscope at $532 \mathrm{~nm}$ excitation.

The NDIR sensor was interfaced to a National Instruments DAQ card (NI USB-6353) to allow for automatic control and data acquisition via LabVIEW@ software. The microheater was voltage modulated by a $2 \mathrm{~Hz}$ periodic squarewave using a custom amplifier. A custom preamplifier ( $60 \mathrm{~dB}$ voltage gain) and software based lock-in amplifier ( $1 \mathrm{~s}$ integration time; $\sim 50 \mathrm{~dB}$ SNR) were used to recover the signal detected by the thermopile from the background noise. The full 16-bits of the A/D conversion were used for the measurement. The overall flow rate employed for the $\mathrm{CO}_{2}$ sensing was $200 \mathrm{sccm}$, achieved using a combination of computer controlled mass flow controllers (MKS).

Received: 1 August 2021; Accepted: 3 November 2021

Published online: 25 November 2021

\section{References}

1. Popa, D. \& Udrea, F. Towards integrated mid-infrared gas sensors. Sensors 19, 2076. https://doi.org/10.3390/s19092076 (2019). 
2. Seymour, F. \& Harris, N. L. Reducing tropical deforestation. Science 365, 756-757. https://doi.org/10.1126/science.aax8546 (2019).

3. Landrigan, P. J. et al. The Lancet Commission on pollution and health. Lancet 391, 462-512. https://doi.org/10.1016/S0140-6736(17) 32345-0 (2018).

4. Barnes, J. H., Chatterton, T. J. \& Longhurst, J. W. Emissions vs exposure: Increasing injustice from road traffic-related air pollution in the United Kingdom. Transp. Res. Part D: Transp. Environ. 73, 56-66. https://doi.org/10.1016/j.trd.2019.05.012 (2019).

5. Morawska, L. et al. Applications of low-cost sensing technologies for air quality monitoring and exposure assessment: How far have they gone?. Environ. Int. 116, 286-299. https://doi.org/10.1016/j.envint.2018.04.018 (2018).

6. Cerchecci, M. et al. A low power iot sensor node architecture for waste management within smart cities context. Sensors 18, 1282. https://doi.org/10.3390/s18041282 (2018).

7. Sun, J. et al. Suspended AlGaN/GaN HEMT NO 2 gas sensor integrated with micro-heater. J. Microelectromech. Syst. 28, 997-1004. https://doi.org/10.1109/JMEMS.2019.2943403 (2019).

8. Non-dispersive Infrared (NDIR) Market. Tech. Rep., MarketsandMarkets (2020).

9. Fujita, H. et al. AlInSb mid-infrared LEDs of high luminous efficiency for gas sensors. Phys. Stat. Solidi (A) 215, 1700449. https:// doi.org/10.1002/pssa.201700449 (2018).

10. Udrea, F. \& Luca, A. D. CMOS technology platform for ubiquitous microsensors. In 2017 International Semiconductor Conference (CAS), 43-52. https://doi.org/10.1109/SMICND.2017.8101149 (2017).

11. De Luca, A. et al. Enhanced spectroscopic gas sensors using in-situ grown carbon nanotubes. Appl. Phys. Lett. 106, 194101. https:// doi.org/10.1063/1.4921170(2015).

12. Santra, S. et al. Post-CMOS wafer level growth of carbon nanotubes for low-cost microsensors-a proof of concept. Nanotechnology 21, 485301. https://doi.org/10.1088/0957-4484/21/48/485301 (2010).

13. Pusch, A. et al. A highly efficient CMOS nanoplasmonic crystal enhanced slow-wave thermal emitter improves infrared gas-sensing devices. Sci. Rep. 5, 17451. https://doi.org/10.1038/srep17451 (2015).

14. Lochbaum, A. et al. On-chip narrowband thermal emitter for mid-IR optical gas sensing. ACS Photon. 4, 1371-1380. https://doi. org/10.1021/acsphotonics.6b01025 (2017).

15. Kaul, A. B., Coles, J. B., Eastwood, M., Green, R. O. \& Bandaru, P. R. Ultra-high optical absorption efficiency from the ultraviolet to the infrared using multi-walled carbon nanotube ensembles. Small 9, 1058-1065. https://doi.org/10.1002/smll.201202232 (2013).

16. Mizuno, K. et al. A black body absorber from vertically aligned single-walled carbon nanotubes. Proc. Natl. Acad. Sci. 106, 6044-6047. https://doi.org/10.1073/pnas.0900155106 (2009).

17. Bom, D. et al. Thermogravimetric analysis of the oxidation of multiwalled carbon nanotubes: Evidence for the role of defect sites in carbon nanotube chemistry. Nano Lett. 2, 615-619. https://doi.org/10.1021/nl020297u (2002).

18. Singh, D. K., Iyer, P. K. \& Giri, P. K. Diameter dependence of oxidative stability in multiwalled carbon nanotubes: Role of defects and effect of vacuum annealing. J. Appl. Phys. 108, 084313. https://doi.org/10.1063/1.3491022 (2010).

19. Ali, S. Z. et al. A low-power, low-cost infra-red emitter in CMOS technology. IEEE Sens. J. 15, 6775-6782. https://doi.org/10.1109/ JSEN.2015.2464693 (2015)

20. Reichl, H. \& Grosser, V. Overview and development trends in the field of MEMS packaging. In Technical Digest. MEMS 2001. 14th IEEE International Conference on Micro Electro Mechanical Systems (Cat. No.01CH37090), 1-5. https://doi.org/10.1109/MEMSYS. 2001.906464 (2001).

21. Esashi, M. Wafer level packaging of MEMS. J. Micromech. Microeng. 18, 073001. https://doi.org/10.1088/0960-1317/18/7/073001 (2008).

22. Santra, S., Guha, P. K., Ali, S. Z., Haneef, I. \& Udrea, F. Silicon on insulator diode temperature sensor-a detailed analysis for ultra-high temperature operation. IEEE Sens. J. 10, 997-1003. https://doi.org/10.1109/JSEN.2009.2037822 (2010).

23. Terranova, M., Sessa, V. \& Rossi, M. The world of carbon nanotubes: An overview of CVD growth methodologies. Chem. Vap. Deposition 12, 315-325. https://doi.org/10.1002/cvde.200600030 (2006).

24. Wirth, C. T., Zhang, C., Zhong, G., Hofmann, S. \& Robertson, J. Diffusion- and reaction-limited growth of carbon nanotube forests. ACS Nano 3, 3560-3566. https://doi.org/10.1021/nn900613e (2009).

25. Nasiri, A. \& Ang, S. Application of alumina-based ceramic paste for high-temperature electronics packaging. J. Electron. Packag. 143, 20902. https://doi.org/10.1115/1.4049292 (2021)

26. Golim, O. P. et al. Synthesis of alumina ceramic encapsulation for self-healing materials on thermal barrier coating. J. Phys: Conf. Ser. 985, 012036. https://doi.org/10.1088/1742-6596/985/1/012036 (2018).

27. Musil, J. et al. Thermal stability of alumina thin films containing $\gamma-\mathrm{Al}_{2} \mathrm{O}_{3}$ phase prepared by reactive magnetron sputtering. Appl. Surf. Sci. 257, 1058-1062. https://doi.org/10.1016/j.apsusc.2010.07.107 (2010).

28. Arun Kumar, D., Merline Shyla, J. \& Xavier, F. P. Synthesis and characterization of $\mathrm{TiO}_{2} / \mathrm{SiO}_{2}$ nano composites for solar cell applications. Appl. Nanosci. 2, 429-436. https://doi.org/10.1007/s13204-012-0060-5 (2012).

29. Popa, D., Ali, S. Z., Hopper, R., Dai, Y. \& Udrea, F. Smart CMOS mid-infrared sensor array. Opt. Lett. 44, 4111. https://doi.org/10. 1364/OL.44.004111 (2019).

30. Dresselhaus, M., Dresselhaus, G., Saito, R. \& Jorio, A. Raman spectroscopy of carbon nanotubes. Phys. Rep. 409, 47-99. https:// doi.org/10.1016/j.physrep.2004.10.006 (2005).

31. Brand, O. \& Fedder, G. K. (eds) CMOS-MEMS (aaWILEY-VCH, 2005).

\section{Acknowledgements}

We acknowledge funding from EPSRC (EP/S031847/1, EP/S030247/1, EP/P005152/1). V.-P. V.-R. acknowledges EPSRC Doctoral Training Award (EP/M508007/1) and support from NPL, and J.A.W. acknowledges the support of his Royal Society Dorothy Hodgkin Research Fellowship.

\section{Author contributions}

D.P. and F.U. conceived the idea. D.P. and R.H. designed the project. D.P., R.H., S.Z.A. and M.T.C. fabricated the devices. D.P., R.H., Y.F., V.-P. V.-R., R.C. and J.N. characterised the devices. D.P. and R.H. tested the devices. D.P., R.H., Y.X., J.W.G. and F.U. analysed the results. D.P. supervised the work and wrote the manuscript. All authors reviewed the manuscript.

\section{Competing interests}

The authors declare no competing interests.

\section{Additional information}

Correspondence and requests for materials should be addressed to D.P.

Reprints and permissions information is available at www.nature.com/reprints. 
Publisher's note Springer Nature remains neutral with regard to jurisdictional claims in published maps and institutional affiliations.

(c) (1) Open Access This article is licensed under a Creative Commons Attribution 4.0 International License, which permits use, sharing, adaptation, distribution and reproduction in any medium or format, as long as you give appropriate credit to the original author(s) and the source, provide a link to the Creative Commons licence, and indicate if changes were made. The images or other third party material in this article are included in the article's Creative Commons licence, unless indicated otherwise in a credit line to the material. If material is not included in the article's Creative Commons licence and your intended use is not permitted by statutory regulation or exceeds the permitted use, you will need to obtain permission directly from the copyright holder. To view a copy of this licence, visit http://creativecommons.org/licenses/by/4.0/.

(C) The Author(s) 2021 